\title{
Design of an ergonomic ultrasound system: accommodation of user anthropometrics
}

\author{
Sung Park ${ }^{*}$, Jinho Yim and Goeun Lee \\ User Experience Design Group, Digital Media \& Communications R\&D Center, Samsung Electronics
}

\begin{abstract}
Long-term use of medical imaging devices requires significant improvements to the user experience. One factor that impact upon such experience is whether the device is ergonomically built, ecologically designed, and leverages the current medical practice. In this research, we took a holistic and systematic approach to design an effective and biomechanically-fit ultrasound system. Research methods from behavior science (e.g., contextual inquiry, pseudo experiments) had been adopted to involve the users (sonographers) early in the design process. The end results - product design guideline for a cart type ultrasound system and control panel layout - were reviewed by the users and adjusted so that the design is within the range of an acceptable learning curve while maintaining innovativeness, a differentiated value over competitor's ultrasound devices.
\end{abstract}

Keywords: antropometrics, biomechanics, contextual inquiry, ergonomics, medical device, product design, user experience, user research, system design

\section{Introduction}

Musculoskeletal symptoms are pervasive among sonographers and doctors who use ultrasound systems in their medical practice. Our observation with sonographers indicated that neck and back problems are the most obtrusive. Specifically, twisting/bending the neck and torso, non-neutral postures of the shoulder, and applying pressure with the transducer with musculoskeletal discomfort are the major disorders identified by our interview and supported by the literature [1].

To reduce musculoskeletal disorders and to increase work efficiency, accommodating user anthropometrics to the design of ultrasound systems is essential. However, our competitive analysis proved that although competitors claim that ergonomic features are built in their systems, many measures of the system failed to comply with standard biometric guidelines. Sonographers also echoed this status quo in many instances where misdesigned features led not only to discomfort but to a behavior slip or a task error during an ultrasonic diagnosis.
We therefore established a strict product design guideline, which includes the control panel design, based on user anthropometrics, considering both the upper and lower end of user population. To achieve ecological validity, we produced a task flow diagram by observing live diagnosis sessions and analyzed the work environment to consider all aspects of ultrasonic medical practice (e.g., height of patient's bed, arm movement and reach of a sonographer) and adjusted our guideline accordingly.

In this paper, we discuss how ergonomic usercentered design principles were applied to the design of a cart type ultrasound system. In particular, we discuss how we produced the product design guideline (section 2) and the control panel layout (section 3) of an ultrasound imaging device.

\section{Ergonomic product design guideline}

To produce a product design guideline, listing the relevant design items is vital. We had extracted such items by studying competitor's devices and by identifying key variables that seem to cause inconveniency (See Table 1).

*E-mail: sung76.park@samsung.com 

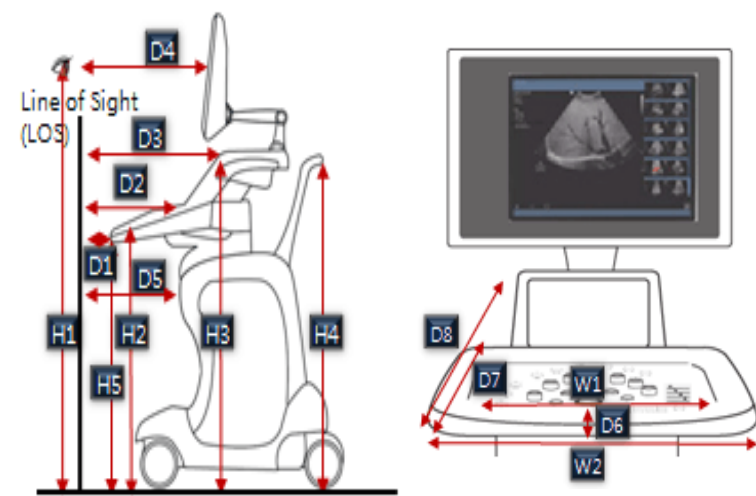

Table 1

Ergonomic product design guideline for a cart type ultrasound system. Guideline is based on 5\%ile biometrics of U.S. population with an assumption of sonographer's chair height being $500 \mathrm{~mm}$.

Only representative measures are reported to confidentiality.

\begin{tabular}{|l|l|l|}
\hline Var. & Description & M/S(mm) \\
\hline H1 & Seated eye height & 1183 \\
\hline H3 & Seated operational height (maximum) & 980 \\
\hline H4 & Elbow height & $\begin{array}{l}982 \sim \\
1056\end{array}$ \\
\hline H5 & Seated knee height & 635 \\
\hline D1 & Space for operation & 50 \\
\hline D3 & Maximum distance for operation - D1 & 590 \\
\hline D8 & Distance to top of touch screen & 516 \\
\hline W2 & Appropriate control panel width & 500 \\
\hline
\end{tabular}

Observation of a live diagnosis session was essential in understanding the basic stance and posture of a sonographer in relation to a patient. We realized that environmental variables such as the height of patient's bed and sonographer's chair also need to be factored in when producing the guideline (See Table 2).

Design items were three folded: reach related items (e.g., seated eye height), ROM (Range of Motion) related items (e.g., appropriate width of operation), and clearance related items (e.g., seated knee height). The proposed appropriate measurements (Table 1) were based on the average of $50 \%$ ile of male's and female's anthropometrics extracted from highly cited references [2][3][4]. Items which minimum measures were required used 95\%tile male population's metrics, and in vice versa, 5\%tile female's. When measures seem to be too extreme - a potential decrement in ecological validity $-25 \%$ tile Korean female's measures were used.

Sonographers appealed that their knees/legs often interfere with the lower body of the device which
Table 2

Appropriate environmental variables to counter muscle stress based on $5 \%$ ile biometrics of U.S. female population. Table 1 was built on this assumption.

\begin{tabular}{|l|l|}
\hline Environmental Variables & Measures (mm) \\
\hline Height of Bed & 470 \\
\hline Patient's Torso Height & 194 \\
\hline Elbow Height & 164 \\
\hline Height of Chair & 500 \\
\hline $\begin{array}{l}\text { Consideration of Individual Differences } \\
\text { in Torso Height }\end{array}$ & $\sim 545$ \\
\hline
\end{tabular}

was confirmed via observation in a live session so we not only provided appropriate space in between (D1, D5) but also made sure the device can maintain its balance as a body to maintain the guideline's feasibility.

Although the appropriate slope of control panels is typically between $15^{\circ}$ to $20^{\circ}, 15^{\circ}$ was recommended because the width of our control panel (W2). To clearly see the touch display and to reduce wrist stress, a slope of $45^{\circ}$ was recommended for the touch display.

We noticed that the competitors failed to comply with a number of design items: the angle/width of the control panel, height from the ground to the control panel, etc. Without doubt, designing the system within the guideline's projected design space would achieve differentiated customer value.

\section{Control panel layout design}

Four steps were conducted to design the control panel layout: 1) Task Analysis, 2) Function identification, 3) Competitive analysis, 4) Pseudo experiments on the level of inconvenience and the location of keyboard, and 5) Control (Physical User Interface) allocation.

\subsection{Task analysis}

Understanding the task flow of a typical ultrasound session is critical to design an effective user experience. We visited 5+ hospitals, observed 50+ ultrasound live session, and conducted $10+$ in-depth interviews with doctors, sonographers, and nurses. Although not written to describe ultrasound procedures, competitor's manuals were also helpful in extracting knowledge regarding goals, steps, and processes. 
A task flow diagram was produced to produce a typical journal of a patient as well as the identification of pain points for use in the subsequent design process (See Figure 1).

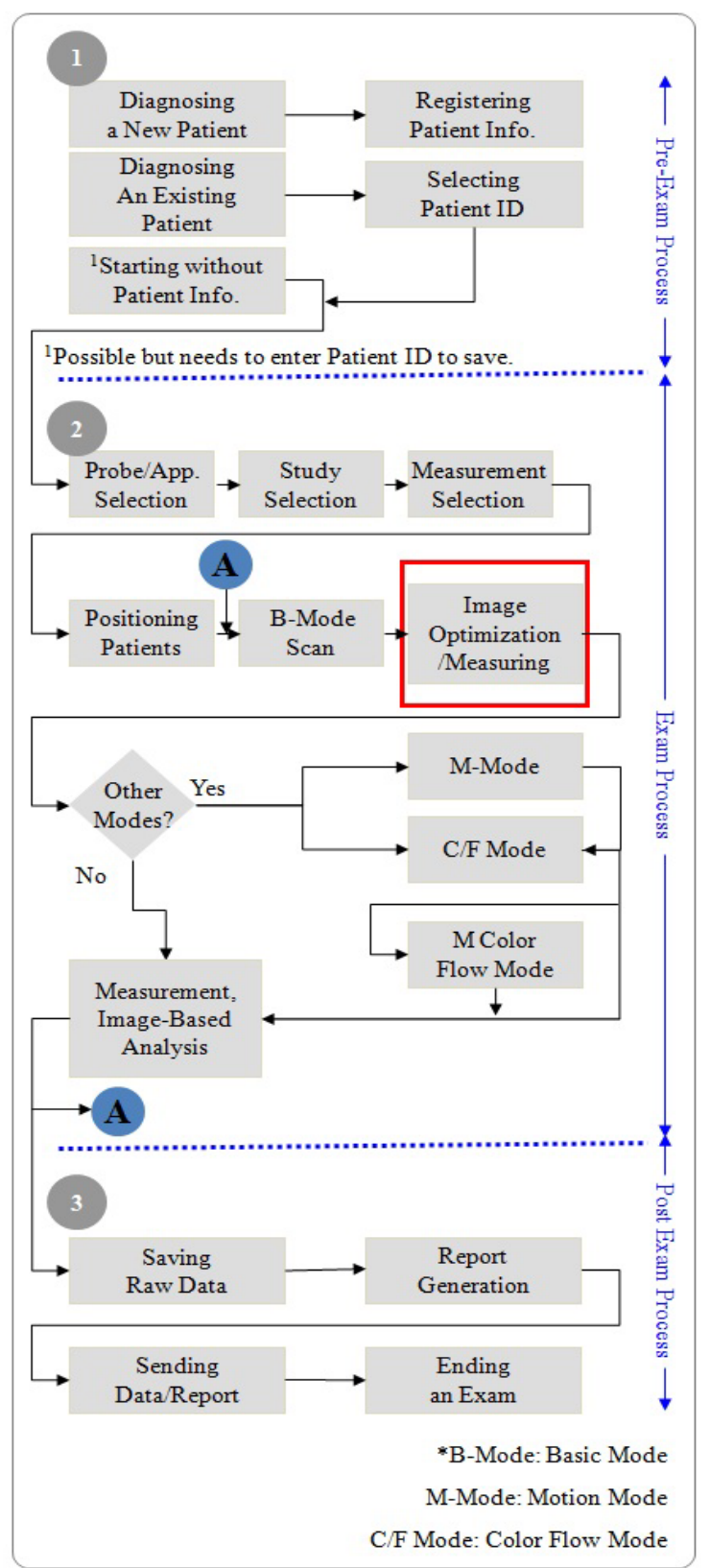

Figure 1. A representative task flow diagram of a live ultrasound diagnosis session.

A usual live session consists of pre-exam process where patient information is entered and selected, followed by examination process where patients are diagnosed. Manipulation of the ultrasound images as well as the measurement them are executed. Images are captured and saved when necessary and reports are written.

We noticed variances in live sessions between different applications (e.g., abdominal vs OB/GYN) and different hospitals (e.g., university hospitals vs private practices) so such differences were embraced in our design. While tasks in ultrasound exams have similarities to other image-based devices (e.g., X-ray, MRI, endoscope), a few characteristics are unique (e.g., the existence of image buffer and the navigation via a trackball to select the right image) and were reflected in our design.

\subsection{Function identification}

General ultrasound systems include an overwhelming number of functions yet the frequency of use varies significantly. Therefore, identifying functions of a cart type ultrasound system is a prerequisite to subsequent design processes. In our interviews, we specifically asked for major functions they operate on as well as their frequency of use. This was supplemented with observations of live diagnosis sessions with real patients in addition to the extraction of function lists from competitor's devices.

The definition and labeling of functions in competitor's devices differ to a great degree so we selected representative functions and label them with agreeable names. We grouped such initial function list into 1) Frequency of use and 2) Function similarity as the following (see Figure 2).

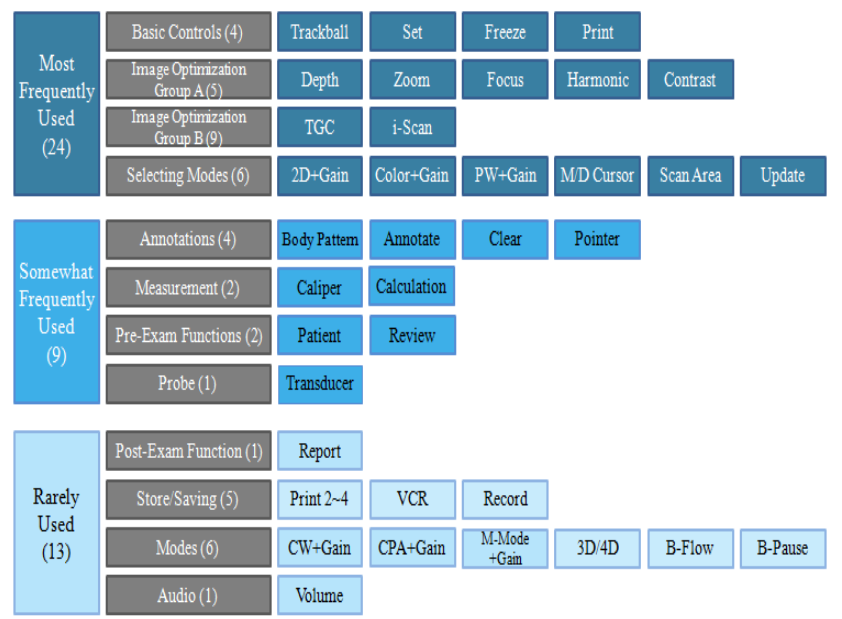

Figure 2. Function extraction grouped by frequency of use and relevancy among functions 
Frequently used functions include trackball manipulation to measure the lesion size and set button to change modes. A repetitive sequence of freeze $\rightarrow$ print has been observed repetitively, consistent with what we had expected via task analysis.

\subsection{Competitive analysis}

Once functions were identified, we mapped functions of devices by major competitors to a blank radiated control panel layout. Competitors' ultrasound systems included Philips iU22, HDI 5000, and General Electronics LOGIQ. We found most buttons, but not all, that were identified as frequently used (in Function identification) were located at area A (see Figure 3) whereas buttons identified as less important were located at area $\mathrm{C}$.

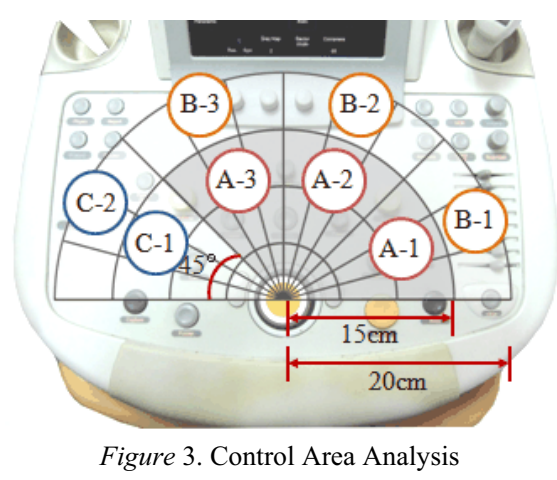

This observation was somewhat consistent with our expectation. Because sonographers typically rest their wrist beneath the trackball and have their patients positioned to their right, it is reasonable to conclude that area $\mathrm{A}$ is more convenient to reach and control than area $\mathrm{B}$ and $\mathrm{C}$. Area $\mathrm{C}$ requires a reverse movement of sonographer's palm and hence produces an uncomfortable flexion.

This muscle stress becomes salient because an ultrasound diagnosis session is a repetitive automatic activity where doctors' left hand manipulates the control rhythmically without visually confirming the buttons before pressing them and with eyes fixed to the monitor. Due to this automaticity, the diagnosis becomes inherently autonomous, involuntary, and unconscious [5][6][7] and requires a thorough investigation of such inconvenience.

\subsection{Level of inconvenience experiment}

An experiment was conducted to measure the level of inconvenience of each partition of the control panel as the participant conducts a mock ultrasound diagnosis. The purpose of the experiment is not only to validate our expectation but also to gain a further partition and prioritization of the control panel's design space so that we can allocate buttons effectively.

To achieve ecologically valid results, we sought to have the experiment setting as close as possible to the medical practice. Observation of live diagnosis sessions suggest patients are not fixated to a single position but rather move their torso when sonographers ask depending on the area of scan. For example, an abdominal scan requires scanning patients' abdominal in between area A and B in Figure 4 whereas a cardiac scan starts from the end of the rib toward the heart (area C) by pressuring the transducer upwards. In general, Sonographers ask patients to respire by breathing in and out or to move patient's torso so that they can acquire a visible scan without noise especially in organs such as kidney, liver, and appendix.

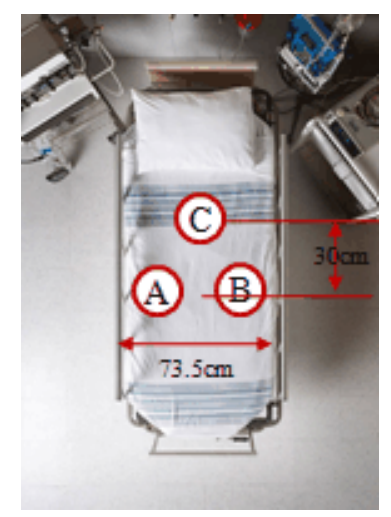

Figure 4. Experiment Task Design. Participants assumed the role of a sonographer and conducted pseudo scanning tasks on three distinct locations.

Experiment consists of four participants in the research team. They conducted a series of mock tasks including representative tasks from pre-screening phase (e.g., entering patient data), screening phase (e.g., B-mode scan), and post-screening phase (e.g., reviewing stored images) with a Philips iU-22. We used the average bed size in hospitals $(73.5 \mathrm{~cm}$ width $)$ and length of patients' abdominal (30cm depth). Based on our control panel design guideline (See Section 2), a radiated control panel layout with a horizontal width of $500 \mathrm{~mm}$ was used with an interval of 
$50 \mathrm{~mm}$ between circular arcs. The radiated layout was divided into 12 areas which were cross-matched with the bed's three areas, resulting in 36 use-cases for the experiment. Subjective responses on perceived inconvenience of each button on a scale of one (most convenient) to seven (most inconvenient) were mapped to the radiated partition layout (see Figure 5).

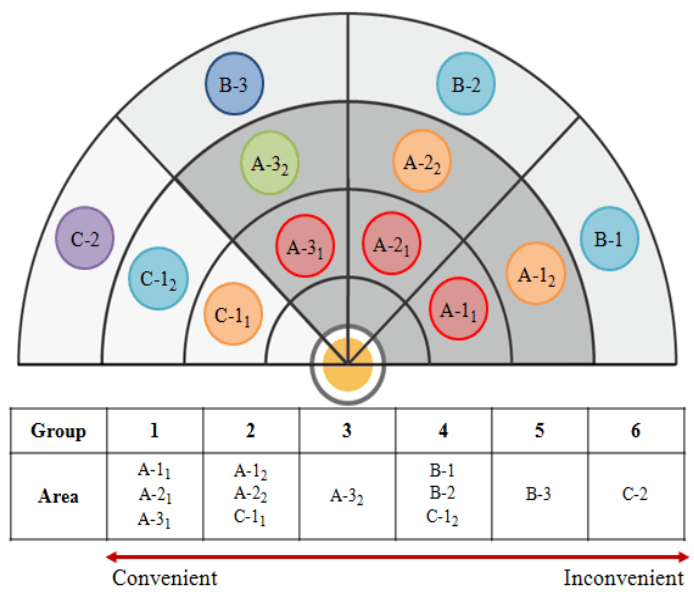

Figure 5. Control panel area partition. Areas were grouped into six groups by the level of inconvenience.

The results not only confirmed our hypothesis but also provided a detailed schema of order by convenience. For example, we now have knowledge that $\mathrm{C}$ $1_{1}$ is actually comfortable to operate at than A- $3_{2}$ because the shorter distance from the trackball $\left(\mathrm{C}-1_{1}\right)$ offsets the flexion due to reverse-movement of the left hand $\left(\mathrm{A}-3_{2}\right)$.

\subsection{Location of keyboard experiment}

Keyboards are used in an ultrasound system to type in patient's demographic information or for hotkeys mapped to certain functions. The location of keyboard is one of the key design decisions that had to be made prior to allocating buttons because it occupies the bulk of the design space. Three keyboard locations were considered: lower left, upper center, and lower center (See Figure 6). Experiment tasks consist of screening behavior with a transducer scanning either location A (proximal) or location B (distal). Typing keys at a report screen was included which does not require a transducer and should have the two hands occupied at the keyboard.
Subjective responses on perceived inconvenience of each pair (the location of keyboard, the type of task) were measured on a scale of one (most convenient) to seven (most inconvenient). Results indicated that in general, lower center location is the most convenient. Upper center was difficult to use when screening - using a transducer - even at a proximal distance and extremely difficult to use at a distal location. Lower left was also difficult when the transducer was located at the far right (location B).

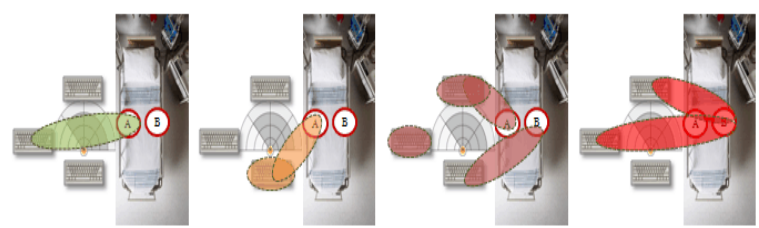

\begin{tabular}{|c|c|c|c|c|}
\hline Group & 1 & 2 & 3 & 4 \\
\hline Section & Lower Left-Proximal & $\begin{array}{c}\text { Lower Center-Report } \\
\text { Lower Center-Proximal }\end{array}$ & $\begin{array}{c}\text { Upper Center-Proximal } \\
\text { Lower Cente-Distal } \\
\text { Upper Center-Report } \\
\text { Lower Left-Report }\end{array}$ & $\begin{array}{c}\text { Upper Center-Distal } \\
\text { Lower Left-Distal }\end{array}$ \\
\hline \multicolumn{2}{|c|}{ Convenient }
\end{tabular}

Figure 6. Keyboard location experiment. Three keyboard locations were tested with the left hand on the keyboard and the right hand on a transducer on two extreme locations (proximal and distal).

\subsection{Control allocation}

Thus far, we have listed functions by importance and the frequency of use (in Function Identification) and divided the radiation space by the level of convenience (in Inconvenience Experiment). We then inter-mapped the functions to the radiated space, allocating the most important and frequently used functions to the most convenient area (See Figure 7). Relevancy among controls was also considered. For example, non-screening functions were grouped at the upper left (e.g., Patient, Transducer, Report) and mode selection functions were grouped at two adjacent rows. In addition, task sequence among buttons was taken into account. For example, because entering patient information precedes selecting transducers/applications/settings, the Patient button was placed on top of the Transducer button. 


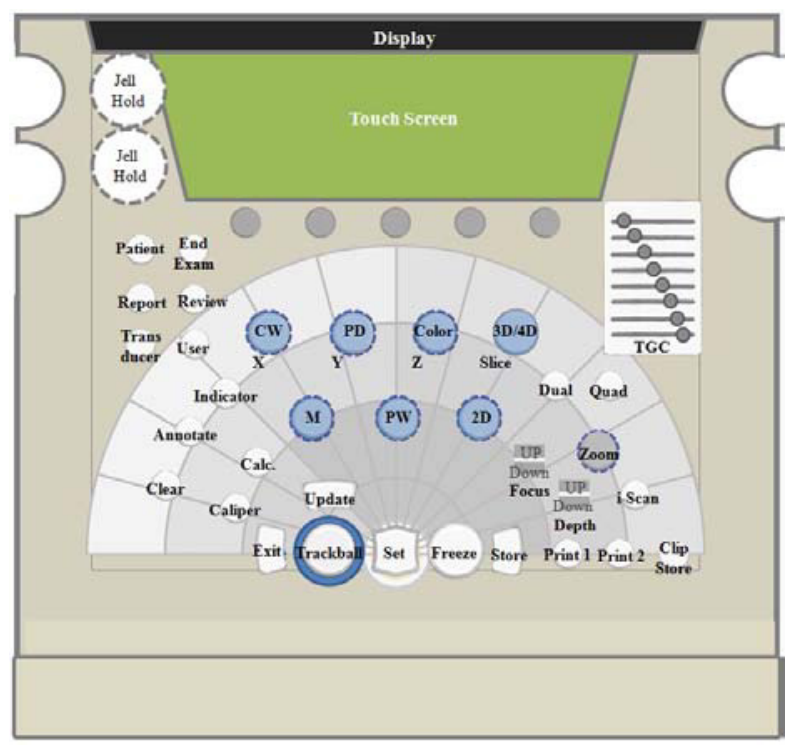

Figure 7. One alternative control panel layout. Based on anthropometric control design guideline [2], minimal distance between buttons was kept at $10 \mathrm{~mm}$ while the size of specific control types were guided as the following: Trackball (50mm), Push buttons (20mm), Rotary push buttons $(24 \mathrm{~mm})$, Set/Exit/Update buttons (20mm).

Prior to the final layout, five alternative layouts were proposed with different emphasis. Figure 7 is one of the five alternative layouts. Two focus group interviews were conducted with sonographers with a paper mockup. Valuable feedbacks were acquired and applied to the final layout. For example, grouping of buttons was fine-tuned, striking a balance between the different needs of OB/GYN and radiology because our target market encompassed both fields. User button was added because of the flexibility and customizability the sonographers asked for. Finally, we would like to note that the final layout is excluded in this paper due to confidentiality.

\section{Discussion and conclusion}

Eighty percent of sonographers have experienced musculoskeletal discomfort during their career [8]. Clearly, ergonomic product design is required to combat the occurrence of musculoskeletal symptoms. In this research, we established a firm product design guideline for a cart type ultrasound system, including the control panel layout. User anthropometrics were integrated with contextual user research from the early stage of the design process by continuously conversing with the doctors and sonographers to maintain robust feasibility and to fulfill iterative usercentered design philosophy.

\section{References}

[1] Albin, T. J., A Comparison of Six Ultrasound Systems with Regard to Conformance to the Industry Standards for the Prevention of Work-Related Musculoskeletal Disorders in Sonography, Ultrasound Benchmark Study, 7, 1-8 (2004).

[2] Department of Defense. Design Criteria for Military Systems, Equipment, and Facilities, MIL-STD-1472F, Military Standard, Human Engineering, (1999).

[3] NRC. Human-System Interface Design Review Guideline, (NUREG-0700, Rev 2), Washington, D.C.: U.S. Nuclear Regulatory Commission, (2002).

[4] Panero, J. and Zelnick, M. Human Dimension and Interior Space: A Source Book of Design Reference Standards, Watson-Guptill, (1979).

[5] Shiffrin, R. and Schneider, W. Controlled and Automatic Human Information Processing, Psychological Review, 84(1), 1-66, (1977).

[6] Schneider, W. and Shiffrin, R. Controlled and Automatic Human Information Processing, Psychological Review, 84(1), 1-66, (1977).

[7] Bargh, J. A. Conditional Automaticity: Varieties of Automatic Influence in Social Perception and Cognition. In J. S. Ulman \& J. A. Bargh (Eds.), Unintended Thought, 3-51. (1989)

[8] Magnavita, N., Bevilacqua, L., Mirk, P., Fileni A., and Castellino, N. Work-related musculoskeletal complaints in sonologists. Journal of Occupational and Environmental Medicine, 11, 981-988 (1999). 